\title{
Structural characterization of
}

\section{WY111403}

Dr. Alexandre F. T. Yokochi

OREGON STATE Department of Chemistry

UNIVERSITY Oregon State University

Phone: (541) 737-6724

Email: alex.yokochi@orst.edu 
Experimental details for WY111403.

The crystalline sample used was that supplied without further work. Determination of the crystallographic parameters, data collection and structure solution and refinement was done as described elsewhere, ${ }^{1}$ with the following details:

A well shaped crystal of dimensions $0.1 \times 0.1 \times 0.05 \mathrm{~mm}^{3}$ was selected and mounted on the tip of a thin glass fiber using a dab of Paratone. Crystal quality evaluation and preliminary indexing were performed from four images of 10 degrees rotation about omega, each separated from the others by 50 degrees. Proving to be a satisfactory crystal, a full set of 80 frames of 10 degrees rotation about omega was collected. The frames were integrated using the determined unit cell, using the program TwinSolve as included in Rigaku/MSC's software package CrystalClear to yield a massively redundant data set of 31794 reflections. Correction for the effects of absorption anisotropy was carried out by means of multiscans ${ }^{2}$ as programmed in TwinSolve. Finally, a data set consisting of 5066 unique reflections in the range (-9 - 9,-15 - 15,-36 - 33) was generated with an R(merge) of 0.080. The reported unit cell was refined using all 2733 reflections with intensities greater than 10 times their e.s.d.s in the range $2.92^{\circ}<\theta<35.92^{\circ}$.

The structure was solved using direct methods as programmed in SHELXS- $90,{ }^{3}$ which revealed the positions of most atoms in the molecule excepting the carbon atoms belonging to the TES group. The solution was refined using the program SHELXL-97, ${ }^{4}$ followed by Fourier synthesis, which revealed the positions of the remaining atoms. Though all hydrogen atoms could be clearly identified from the Fourier map, in order to preserve a favorable data-to-parameter ratio the hydrogen atoms were placed in geometrically idealized positions, with the C$\mathrm{H}$ distance allowed to refine. The hydrogen atoms were given a displacement parameter equal to 1.5 times (methyl group) or 1.2 times (all other hydrogens) the equivalent isotropic displacement parameter of the atom it is attached to. During the final cycle of least squares refinement, all non-hydrogen atoms were refined with anisotropic displacement parameters. The refined value of the absolute structure parameter (Flack parameter) ${ }^{5}$ of $0.02(2)$ indicates that the model obtained accurately depicts the absolute structure of the molecule.

An ORTEP $^{6}$ of the final model is given in Figure 1, with displacement ellipsoids drawn at the $50 \%$ probability level. All unique atoms are labeled, and atoms generated by symmetry are left unlabelled.

\footnotetext{
${ }^{1}$ new reference, probably the 'selenide paper' some journal or another $\mathbf{2 0 0 3}$ volume number, page numbers.

${ }^{2}$ Blessing, R. H. Acta Crystallogr., Sect A 1995, 51, 33-38.

${ }^{3}$ Sheldrick, G. M. Acta Crystallogr. 1990, A46, 467.

${ }^{4}$ Sheldrick, G. M. In Crystallographic Computing 6; Flack, H. D., Parkanyi, L., Simon, K., Eds.; Oxford University Press: Oxford, 1993.

${ }^{5}$ Flack, H. D. Acta Crystallogr. 1983, A39, 876-881.

${ }^{6}$ ORTEP-III - Burnett, M. N.; Johnson, C.K., Report ORNL-6895. Oak Ridge National Laboratory, Oak Ridge, Tennessee, 1996. ORTEP3 for Windows - Farrugia, L. J. J. Appl. Crystallogr. 1997, $30,565$.
} 


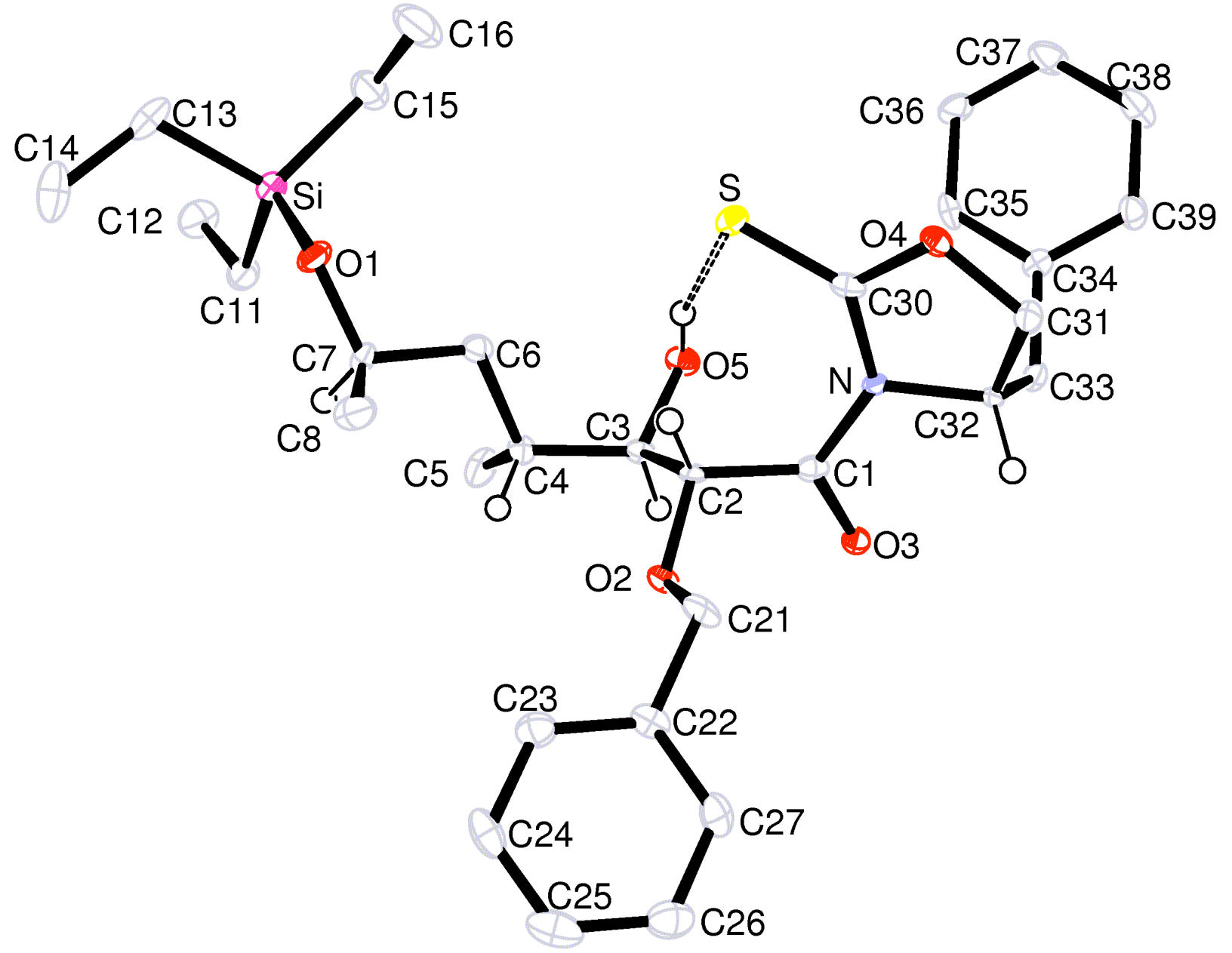

Figure 1: ORTEP with 50\% displacement ellipsoids. 
Table 1. Crystal data and structure refinement for WY111403.

Identification code

Empirical formula

Formula weight

Temperature

Wavelength

Crystal system

Space group

Unit cell dimensions

Volume

Z

Density (calculated)

Absorption coefficient

$\mathrm{F}(000)$

Crystal size

Theta range for data collection

Index ranges

Reflections collected

Independent reflections

Completeness to theta $=68.74^{\circ}$

Absorption correction

Max. and min. transmission

Refinement method

Data / restraints / parameters

Goodness-of-fit on $\mathrm{F}^{2}$

Final R indices [I $>2 \operatorname{sigma}(\mathrm{I})]$

$\mathrm{R}$ indices (all data)

Absolute structure parameter

Largest diff. peak and hole
WY111403

$\mathrm{C}_{31} \mathrm{H}_{45} \mathrm{NO}_{5} \mathrm{SSi}$

571.83

100(2) K

$1.54060 \AA$

Orthorhombic

$\mathrm{P} 2{ }_{1} 2_{1}$

$a=8.0726(4) \AA$

$\alpha=90^{\circ}$.

$\mathrm{b}=12.9005(8) \AA$

$\beta=90^{\circ}$.

$\mathrm{c}=30.1667(15) \AA$

$\gamma=90^{\circ}$.

3141.6(3) $\AA^{3}$

4

$1.209 \mathrm{Mg} / \mathrm{m}^{3}$

$1.585 \mathrm{~mm}^{-1}$

1232

$0.1 \times 0.1 \times 0.05 \mathrm{~mm}^{3}$

2.92 to $71.36^{\circ}$.

$-9<=\mathrm{h}<=9,-15<=\mathrm{k}<=15,-36<=\mathrm{l}<=33$

31794

$5066[\mathrm{R}(\mathrm{int})=0.080]$

$94.6 \%$

Semi-empirical from equivalents

1.0000 and 0.9304

Full-matrix least-squares on $\mathrm{F}^{2}$

5066 / 0 / 359

1.025

$\mathrm{R} 1=0.0512, \mathrm{wR} 2=0.0704$

$\mathrm{R} 1=0.0739, \mathrm{wR} 2=0.0757$

$0.02(2)$

0.278 and -0.229 e. $\AA^{-3}$ 
Table 2. Atomic coordinates ( $\left.\times 10^{4}\right)$ and equivalent isotropic displacement parameters $\left(\AA^{2} \times 10^{3}\right)$ for WY111403. $\mathrm{U}(\mathrm{eq})$ is defined as one third of the trace of the orthogonalized $\mathrm{U}^{\mathrm{ij}}$ tensor.

\begin{tabular}{|c|c|c|c|c|}
\hline & $\mathrm{x}$ & $\mathrm{y}$ & $\mathrm{z}$ & $\mathrm{U}(\mathrm{eq})$ \\
\hline S & $6152(1)$ & $9516(1)$ & $9007(1)$ & $17(1)$ \\
\hline $\mathrm{Si}$ & $306(1)$ & $9396(1)$ & $7665(1)$ & $17(1)$ \\
\hline $\mathrm{O}(1)$ & 1890(3) & $10208(2)$ & $7703(1)$ & $18(1)$ \\
\hline $\mathrm{O}(2)$ & 4713(3) & $12926(2)$ & 9119(1) & $13(1)$ \\
\hline $\mathrm{O}(3)$ & $5223(3)$ & $12216(2)$ & 9964(1) & $16(1)$ \\
\hline $\mathrm{O}(4)$ & $8457(3)$ & $9597(2)$ & $9601(1)$ & $16(1)$ \\
\hline $\mathrm{O}(5)$ & 2727(3) & $10498(2)$ & $9415(1)$ & $16(1)$ \\
\hline $\mathrm{N}$ & $6608(3)$ & $10825(2)$ & $9718(1)$ & $10(1)$ \\
\hline$C(1)$ & $5486(4)$ & $11635(2)$ & $9657(1)$ & $13(1)$ \\
\hline$C(2)$ & 4683(4) & $11838(2)$ & $9205(1)$ & 12(1) \\
\hline $\mathrm{C}(3)$ & 2862(4) & $11522(2)$ & $9233(1)$ & 11(1) \\
\hline $\mathrm{C}(4)$ & 1924(4) & $11653(2)$ & $8795(1)$ & $13(1)$ \\
\hline$C(5)$ & $64(4)$ & $11712(3)$ & $8886(1)$ & $22(1)$ \\
\hline$C(6)$ & 2331(4) & $10797(2)$ & $8460(1)$ & $14(1)$ \\
\hline$C(7)$ & 1966(4) & 11101(3) & 7981(1) & $16(1)$ \\
\hline $\mathrm{C}(8)$ & $3313(4)$ & 11796(3) & 7794(1) & $23(1)$ \\
\hline $\mathrm{C}(11)$ & $-1664(4)$ & $10052(3)$ & $7835(1)$ & $20(1)$ \\
\hline $\mathrm{C}(12)$ & $-3210(4)$ & 9382(3) & $7773(1)$ & $26(1)$ \\
\hline$C(13)$ & $236(5)$ & $9005(3)$ & 7071(1) & $24(1)$ \\
\hline$C(14)$ & $-261(5)$ & $9876(3)$ & $6750(1)$ & $36(1)$ \\
\hline $\mathrm{C}(15)$ & $681(4)$ & $8227(3)$ & $8016(1)$ & $27(1)$ \\
\hline$C(16)$ & $2423(5)$ & $7769(3)$ & $7967(1)$ & $41(1)$ \\
\hline $\mathrm{C}(21)$ & $6360(4)$ & $13303(2)$ & 9039(1) & $18(1)$ \\
\hline$C(22)$ & 6264(4) & $14409(3)$ & $8879(1)$ & $15(1)$ \\
\hline$C(23)$ & $5565(4)$ & $14626(3)$ & $8470(1)$ & $21(1)$ \\
\hline$C(24)$ & $5550(4)$ & $15639(3)$ & $8309(1)$ & $24(1)$ \\
\hline$C(25)$ & $6232(5)$ & $16429(3)$ & $8560(1)$ & $27(1)$ \\
\hline$C(26)$ & 6929(4) & $16218(3)$ & $8966(1)$ & $27(1)$ \\
\hline$C(27)$ & 6941(4) & $15205(3)$ & 9123(1) & $20(1)$ \\
\hline $\mathrm{C}(30)$ & 7066(4) & $10008(2)$ & $9447(1)$ & $13(1)$ \\
\hline $\mathrm{C}(31)$ & $9075(4)$ & $10194(2)$ & $9976(1)$ & $16(1)$ \\
\hline
\end{tabular}




\begin{tabular}{lllll}
$\mathrm{C}(32)$ & $7595(4)$ & $10821(2)$ & $10131(1)$ & $11(1)$ \\
$\mathrm{C}(33)$ & $6647(4)$ & $10374(2)$ & $10527(1)$ & $14(1)$ \\
$\mathrm{C}(34)$ & $6465(4)$ & $9205(2)$ & $10520(1)$ & $11(1)$ \\
$\mathrm{C}(35)$ & $5353(4)$ & $8710(2)$ & $10244(1)$ & $15(1)$ \\
$\mathrm{C}(36)$ & $5275(4)$ & $7636(2)$ & $10233(1)$ & $20(1)$ \\
$\mathrm{C}(37)$ & $6295(4)$ & $7050(3)$ & $10500(1)$ & $26(1)$ \\
$\mathrm{C}(38)$ & $7408(5)$ & $7534(3)$ & $10776(1)$ & $23(1)$ \\
$\mathrm{C}(39)$ & $7491(4)$ & $8611(3)$ & $10790(1)$ & $18(1)$ \\
& & & & \\
\hline
\end{tabular}


Table 3. Bond lengths $[\AA]$ and angles $\left[{ }^{\circ}\right]$ for WY111403.

\begin{tabular}{|c|c|c|c|}
\hline S-C(30) & $1.647(3)$ & $\mathrm{C}(33)-\mathrm{C}(34)$ & $1.516(4)$ \\
\hline $\mathrm{Si}-\mathrm{O}(1)$ & $1.657(2)$ & $\mathrm{C}(34)-\mathrm{C}(35)$ & $1.380(4)$ \\
\hline $\mathrm{Si}-\mathrm{C}(13)$ & $1.860(3)$ & $\mathrm{C}(34)-\mathrm{C}(39)$ & $1.391(4)$ \\
\hline $\mathrm{Si}-\mathrm{C}(15)$ & $1.868(4)$ & $C(35)-C(36)$ & $1.388(4)$ \\
\hline $\mathrm{Si}-\mathrm{C}(11)$ & $1.873(3)$ & $C(36)-C(37)$ & $1.377(4)$ \\
\hline $\mathrm{O}(1)-\mathrm{C}(7)$ & $1.427(4)$ & $\mathrm{C}(37)-\mathrm{C}(38)$ & $1.376(5)$ \\
\hline $\mathrm{O}(2)-\mathrm{C}(2)$ & $1.427(3)$ & $\mathrm{C}(38)-\mathrm{C}(39)$ & $1.392(4)$ \\
\hline $\mathrm{O}(2)-\mathrm{C}(21)$ & $1.437(4)$ & & \\
\hline $\mathrm{O}(3)-\mathrm{C}(1)$ & $1.211(3)$ & $\mathrm{O}(1)-\mathrm{Si}-\mathrm{C}(13)$ & $105.19(14)$ \\
\hline $\mathrm{O}(4)-\mathrm{C}(30)$ & $1.325(4)$ & $\mathrm{O}(1)-\mathrm{Si}-\mathrm{C}(15)$ & $110.20(14)$ \\
\hline $\mathrm{O}(4)-\mathrm{C}(31)$ & $1.458(3)$ & $\mathrm{C}(13)-\mathrm{Si}-\mathrm{C}(15)$ & $109.38(17)$ \\
\hline $\mathrm{O}(5)-\mathrm{C}(3)$ & $1.436(3)$ & $\mathrm{O}(1)-\mathrm{Si}-\mathrm{C}(11)$ & $110.51(13)$ \\
\hline $\mathrm{N}-\mathrm{C}(30)$ & $1.384(4)$ & $\mathrm{C}(13)-\mathrm{Si}-\mathrm{C}(11)$ & $111.18(16)$ \\
\hline $\mathrm{N}-\mathrm{C}(1)$ & $1.395(4)$ & $\mathrm{C}(15)-\mathrm{Si}-\mathrm{C}(11)$ & $110.27(16)$ \\
\hline $\mathrm{N}-\mathrm{C}(32)$ & $1.480(3)$ & $\mathrm{C}(7)-\mathrm{O}(1)-\mathrm{Si}$ & $125.8(2)$ \\
\hline $\mathrm{C}(1)-\mathrm{C}(2)$ & $1.533(4)$ & $\mathrm{C}(2)-\mathrm{O}(2)-\mathrm{C}(21)$ & $112.3(2)$ \\
\hline $\mathrm{C}(2)-\mathrm{C}(3)$ & $1.528(4)$ & $\mathrm{C}(30)-\mathrm{O}(4)-\mathrm{C}(31)$ & $110.5(2)$ \\
\hline $\mathrm{C}(3)-\mathrm{C}(4)$ & $1.530(4)$ & $\mathrm{C}(30)-\mathrm{N}-\mathrm{C}(1)$ & $131.7(3)$ \\
\hline $\mathrm{C}(4)-\mathrm{C}(5)$ & $1.528(4)$ & $\mathrm{C}(30)-\mathrm{N}-\mathrm{C}(32)$ & $110.6(2)$ \\
\hline $\mathrm{C}(4)-\mathrm{C}(6)$ & $1.532(4)$ & $\mathrm{C}(1)-\mathrm{N}-\mathrm{C}(32)$ & $117.6(2)$ \\
\hline $\mathrm{C}(6)-\mathrm{C}(7)$ & $1.527(4)$ & $\mathrm{O}(3)-\mathrm{C}(1)-\mathrm{N}$ & $118.5(3)$ \\
\hline$C(7)-C(8)$ & $1.518(4)$ & $\mathrm{O}(3)-\mathrm{C}(1)-\mathrm{C}(2)$ & $120.0(3)$ \\
\hline $\mathrm{C}(11)-\mathrm{C}(12)$ & $1.530(4)$ & $\mathrm{N}-\mathrm{C}(1)-\mathrm{C}(2)$ & $121.4(3)$ \\
\hline$C(13)-C(14)$ & $1.539(5)$ & $\mathrm{O}(2)-\mathrm{C}(2)-\mathrm{C}(3)$ & $106.7(2)$ \\
\hline$C(15)-C(16)$ & $1.532(5)$ & $\mathrm{O}(2)-\mathrm{C}(2)-\mathrm{C}(1)$ & $108.8(2)$ \\
\hline$C(21)-C(22)$ & $1.508(4)$ & $\mathrm{C}(3)-\mathrm{C}(2)-\mathrm{C}(1)$ & $108.2(3)$ \\
\hline$C(22)-C(27)$ & $1.377(4)$ & $\mathrm{O}(5)-\mathrm{C}(3)-\mathrm{C}(2)$ & $109.8(3)$ \\
\hline$C(22)-C(23)$ & $1.387(4)$ & $\mathrm{O}(5)-\mathrm{C}(3)-\mathrm{C}(4)$ & $113.2(2)$ \\
\hline$C(23)-C(24)$ & $1.394(5)$ & $\mathrm{C}(2)-\mathrm{C}(3)-\mathrm{C}(4)$ & $113.5(2)$ \\
\hline$C(24)-C(25)$ & $1.383(5)$ & $\mathrm{C}(5)-\mathrm{C}(4)-\mathrm{C}(3)$ & $109.7(3)$ \\
\hline$C(25)-C(26)$ & $1.375(4)$ & $\mathrm{C}(5)-\mathrm{C}(4)-\mathrm{C}(6)$ & $111.4(3)$ \\
\hline$C(26)-C(27)$ & $1.390(4)$ & $\mathrm{C}(3)-\mathrm{C}(4)-\mathrm{C}(6)$ & $112.5(3)$ \\
\hline$C(31)-C(32)$ & $1.516(4)$ & $C(7)-C(6)-C(4)$ & $113.5(3)$ \\
\hline$C(32)-C(33)$ & $1.532(4)$ & $\mathrm{O}(1)-\mathrm{C}(7)-\mathrm{C}(8)$ & $106.8(3)$ \\
\hline
\end{tabular}




\begin{tabular}{lllr}
$\mathrm{O}(1)-\mathrm{C}(7)-\mathrm{C}(6)$ & $110.9(3)$ & $\mathrm{O}(4)-\mathrm{C}(30)-\mathrm{S}$ & $120.5(2)$ \\
$\mathrm{C}(8)-\mathrm{C}(7)-\mathrm{C}(6)$ & $111.5(3)$ & $\mathrm{N}-\mathrm{C}(30)-\mathrm{S}$ & $130.6(3)$ \\
$\mathrm{C}(12)-\mathrm{C}(11)-\mathrm{Si}$ & $113.8(2)$ & $\mathrm{O}(4)-\mathrm{C}(31)-\mathrm{C}(32)$ & $104.6(2)$ \\
$\mathrm{C}(14)-\mathrm{C}(13)-\mathrm{Si}$ & $114.7(2)$ & $\mathrm{N}-\mathrm{C}(32)-\mathrm{C}(31)$ & $99.6(2)$ \\
$\mathrm{C}(16)-\mathrm{C}(15)-\mathrm{Si}$ & $113.9(3)$ & $\mathrm{N}-\mathrm{C}(32)-\mathrm{C}(33)$ & $112.9(3)$ \\
$\mathrm{O}(2)-\mathrm{C}(21)-\mathrm{C}(22)$ & $109.1(2)$ & $\mathrm{C}(31)-\mathrm{C}(32)-\mathrm{C}(33)$ & $115.7(2)$ \\
$\mathrm{C}(27)-\mathrm{C}(22)-\mathrm{C}(23)$ & $119.2(3)$ & $\mathrm{C}(34)-\mathrm{C}(33)-\mathrm{C}(32)$ & $114.3(3)$ \\
$\mathrm{C}(27)-\mathrm{C}(22)-\mathrm{C}(21)$ & $120.9(3)$ & $\mathrm{C}(35)-\mathrm{C}(34)-\mathrm{C}(39)$ & $119.1(3)$ \\
$\mathrm{C}(23)-\mathrm{C}(22)-\mathrm{C}(21)$ & $119.8(3)$ & $\mathrm{C}(35)-\mathrm{C}(34)-\mathrm{C}(33)$ & $122.1(3)$ \\
$\mathrm{C}(22)-\mathrm{C}(23)-\mathrm{C}(24)$ & $120.2(3)$ & $\mathrm{C}(39)-\mathrm{C}(34)-\mathrm{C}(33)$ & $118.8(3)$ \\
$\mathrm{C}(25)-\mathrm{C}(24)-\mathrm{C}(23)$ & $119.8(3)$ & $\mathrm{C}(34)-\mathrm{C}(35)-\mathrm{C}(36)$ & $120.4(3)$ \\
$\mathrm{C}(26)-\mathrm{C}(25)-\mathrm{C}(24)$ & $120.3(3)$ & $\mathrm{C}(37)-\mathrm{C}(36)-\mathrm{C}(35)$ & $120.4(3)$ \\
$\mathrm{C}(25)-\mathrm{C}(26)-\mathrm{C}(27)$ & $119.6(3)$ & $\mathrm{C}(38)-\mathrm{C}(37)-\mathrm{C}(36)$ & $119.8(3)$ \\
$\mathrm{C}(22)-\mathrm{C}(27)-\mathrm{C}(26)$ & $121.0(3)$ & $\mathrm{C}(37)-\mathrm{C}(38)-\mathrm{C}(39)$ & $120.2(3)$ \\
$\mathrm{O}(4)-\mathrm{C}(30)-\mathrm{N}$ & $108.9(3)$ & $\mathrm{C}(34)-\mathrm{C}(39)-\mathrm{C}(38)$ & $120.2(3)$ \\
& & & \\
\hline
\end{tabular}

Symmetry transformations used to generate equivalent atoms: 
Table 4. Anisotropic displacement parameters $\left(\AA^{2} \times 10^{3}\right)$ for WY111403. The anisotropic displacement factor exponent takes the form: $-2 \pi^{2}\left[h^{2} a^{* 2} U^{11}+\ldots+2 h k a^{*} b^{*} U^{12}\right]$

\begin{tabular}{|c|c|c|c|c|c|c|}
\hline & $\mathrm{U}^{11}$ & $\mathrm{U}^{22}$ & $\mathrm{U}^{33}$ & $\mathrm{U}^{23}$ & $\mathrm{U}^{13}$ & $\mathrm{U}^{12}$ \\
\hline $\mathrm{S}$ & $20(1)$ & $16(1)$ & $16(1)$ & $-5(1)$ & $-2(1)$ & $2(1)$ \\
\hline $\mathrm{Si}$ & $20(1)$ & $16(1)$ & $15(1)$ & $-2(1)$ & $-3(1)$ & $0(1)$ \\
\hline $\mathrm{O}(1)$ & $21(1)$ & $17(1)$ & $17(1)$ & $-7(1)$ & $-2(1)$ & $0(1)$ \\
\hline $\mathrm{O}(2)$ & 11(1) & $10(1)$ & $18(1)$ & $3(1)$ & $0(1)$ & $1(1)$ \\
\hline $\mathrm{O}(3)$ & 19(1) & $14(1)$ & $16(1)$ & $-4(1)$ & $-5(1)$ & $7(1)$ \\
\hline $\mathrm{O}(4)$ & $13(1)$ & $15(1)$ & $19(1)$ & $-1(1)$ & $-2(1)$ & $6(1)$ \\
\hline $\mathrm{O}(5)$ & $18(1)$ & $13(1)$ & $16(1)$ & $3(1)$ & $4(1)$ & $-1(1)$ \\
\hline $\mathrm{N}$ & $10(1)$ & $8(1)$ & $11(1)$ & $-2(1)$ & $-1(1)$ & $0(1)$ \\
\hline$C(1)$ & $11(2)$ & $11(2)$ & $17(2)$ & $-1(1)$ & $2(1)$ & $-2(2)$ \\
\hline$C(2)$ & $13(2)$ & $7(2)$ & $15(2)$ & $-3(1)$ & $0(2)$ & $4(2)$ \\
\hline$C(3)$ & $10(2)$ & $9(2)$ & $15(2)$ & 1(1) & $2(1)$ & $-2(1)$ \\
\hline$C(4)$ & $10(2)$ & $16(2)$ & $13(2)$ & $-1(1)$ & $1(1)$ & $3(2)$ \\
\hline$C(5)$ & $16(2)$ & $30(2)$ & $18(2)$ & $-6(2)$ & $-1(2)$ & $-1(2)$ \\
\hline$C(6)$ & $13(2)$ & $13(2)$ & $18(2)$ & $0(1)$ & $0(1)$ & $1(2)$ \\
\hline$C(7)$ & $20(2)$ & $18(2)$ & $11(2)$ & $-4(1)$ & $-4(2)$ & $0(2)$ \\
\hline$C(8)$ & $30(2)$ & $19(2)$ & $21(2)$ & $-2(2)$ & $4(2)$ & $-3(2)$ \\
\hline $\mathrm{C}(11)$ & $18(2)$ & $23(2)$ & $20(2)$ & $-5(2)$ & $-3(2)$ & $4(2)$ \\
\hline$C(12)$ & $19(2)$ & $29(2)$ & $31(2)$ & $-6(2)$ & $-1(2)$ & $-1(2)$ \\
\hline$C(13)$ & $21(2)$ & $33(2)$ & $19(2)$ & $-13(2)$ & $4(2)$ & $-9(2)$ \\
\hline$C(14)$ & $32(2)$ & $61(3)$ & $16(2)$ & $3(2)$ & $-2(2)$ & $-3(2)$ \\
\hline$C(15)$ & $27(2)$ & $22(2)$ & $32(2)$ & $6(2)$ & $-7(2)$ & $-1(2)$ \\
\hline$C(16)$ & $39(3)$ & $25(3)$ & $58(3)$ & $9(2)$ & $-13(2)$ & $7(2)$ \\
\hline$C(21)$ & $6(2)$ & $14(2)$ & $32(2)$ & $6(2)$ & $0(2)$ & $4(2)$ \\
\hline$C(22)$ & $8(2)$ & $16(2)$ & $22(2)$ & $4(2)$ & $7(1)$ & $0(2)$ \\
\hline$C(23)$ & $22(2)$ & $18(2)$ & $23(2)$ & $0(2)$ & $2(2)$ & $-4(2)$ \\
\hline$C(24)$ & $23(2)$ & $32(2)$ & $17(2)$ & $13(2)$ & $4(2)$ & $5(2)$ \\
\hline$C(25)$ & $25(2)$ & $17(2)$ & $37(2)$ & $8(2)$ & $16(2)$ & $-4(2)$ \\
\hline$C(26)$ & $25(2)$ & $21(2)$ & $35(2)$ & $-2(2)$ & $1(2)$ & $-4(2)$ \\
\hline$C(27)$ & $11(2)$ & $27(2)$ & $23(2)$ & $5(2)$ & $-2(2)$ & $0(2)$ \\
\hline$C(30)$ & $16(2)$ & $8(2)$ & $15(2)$ & $4(1)$ & $6(2)$ & $-2(2)$ \\
\hline $\mathrm{C}(31)$ & $15(2)$ & $16(2)$ & $16(2)$ & $0(1)$ & $-4(1)$ & $-1(2)$ \\
\hline
\end{tabular}




\begin{tabular}{lllllll}
$\mathrm{C}(32)$ & $13(2)$ & $5(2)$ & $14(2)$ & $0(1)$ & $-6(1)$ & $0(1)$ \\
$\mathrm{C}(33)$ & $16(2)$ & $16(2)$ & $11(2)$ & $-3(1)$ & $-3(1)$ & $1(2)$ \\
$\mathrm{C}(34)$ & $12(2)$ & $14(2)$ & $9(1)$ & $0(1)$ & $5(1)$ & $-3(1)$ \\
$\mathrm{C}(35)$ & $11(2)$ & $22(2)$ & $12(2)$ & $6(1)$ & $-2(2)$ & $3(2)$ \\
$\mathrm{C}(36)$ & $16(2)$ & $15(2)$ & $28(2)$ & $-5(2)$ & $-4(2)$ & $-6(2)$ \\
$\mathrm{C}(37)$ & $20(2)$ & $13(2)$ & $44(2)$ & $5(2)$ & $-6(2)$ & $-3(2)$ \\
$\mathrm{C}(38)$ & $16(2)$ & $21(2)$ & $32(2)$ & $11(2)$ & $-2(2)$ & $2(2)$ \\
$\mathrm{C}(39)$ & $14(2)$ & $19(2)$ & $20(2)$ & $3(2)$ & $-4(2)$ & $-2(2)$ \\
& & & & & & \\
\hline
\end{tabular}


Table 5. Hydrogen coordinates ( $\left.\times 10^{4}\right)$ and isotropic displacement parameters $\left(\AA^{2} \times 10^{3}\right)$ for WY111403.

\begin{tabular}{|c|c|c|c|c|}
\hline & $\mathrm{x}$ & $\mathrm{y}$ & $\mathrm{z}$ & $\mathrm{U}(\mathrm{eq})$ \\
\hline HO5 & 3358 & 10107 & 9283 & 23 \\
\hline $\mathrm{H}(2)$ & 5255 & 11454 & 8970 & 14 \\
\hline $\mathrm{H}(3)$ & 2340 & 11993 & 9446 & 13 \\
\hline $\mathrm{H}(4)$ & 2264 & 12316 & 8665 & 15 \\
\hline $\mathrm{H}(5 \mathrm{~A})$ & -312 & 11060 & 9002 & 32 \\
\hline $\mathrm{H}(5 \mathrm{~B})$ & -512 & 11861 & 8615 & 32 \\
\hline $\mathrm{H}(5 \mathrm{C})$ & -151 & 12250 & 9098 & 32 \\
\hline $\mathrm{H}(6 \mathrm{~A})$ & 3495 & 10621 & 8486 & 17 \\
\hline $\mathrm{H}(6 \mathrm{~B})$ & 1693 & 10184 & 8534 & 17 \\
\hline $\mathrm{H}(7)$ & 903 & 11467 & 7969 & 19 \\
\hline $\mathrm{H}(8 \mathrm{~A})$ & 4351 & 11434 & 7797 & 35 \\
\hline $\mathrm{H}(8 \mathrm{~B})$ & 3399 & 12412 & 7971 & 35 \\
\hline $\mathrm{H}(8 \mathrm{C})$ & 3040 & 11982 & 7494 & 35 \\
\hline $\mathrm{H}(11 \mathrm{~A})$ & -1579 & 10247 & 8145 & 24 \\
\hline $\mathrm{H}(11 \mathrm{~B})$ & -1790 & 10683 & 7664 & 24 \\
\hline $\mathrm{H}(12 \mathrm{~A})$ & -3312 & 9191 & 7467 & 39 \\
\hline $\mathrm{H}(12 \mathrm{~B})$ & -4172 & 9768 & 7862 & 39 \\
\hline $\mathrm{H}(12 \mathrm{C})$ & -3118 & 8769 & 7951 & 39 \\
\hline $\mathrm{H}(13 \mathrm{~A})$ & 1320 & 8748 & 6987 & 29 \\
\hline $\mathrm{H}(13 \mathrm{~B})$ & -544 & 8438 & 7040 & 29 \\
\hline $\mathrm{H}(14 \mathrm{~A})$ & -1382 & 10083 & 6808 & 54 \\
\hline $\mathrm{H}(14 \mathrm{~B})$ & -175 & 9630 & 6450 & 54 \\
\hline $\mathrm{H}(14 \mathrm{C})$ & 464 & 10459 & 6789 & 54 \\
\hline $\mathrm{H}(15 \mathrm{~A})$ & 504 & 8411 & 8324 & 32 \\
\hline $\mathrm{H}(15 \mathrm{~B})$ & -125 & 7699 & 7939 & 32 \\
\hline $\mathrm{H}(16 \mathrm{~A})$ & 2616 & 7593 & 7662 & 61 \\
\hline $\mathrm{H}(16 \mathrm{~B})$ & 2515 & 7157 & 8146 & 61 \\
\hline $\mathrm{H}(16 \mathrm{C})$ & 3228 & 8270 & 8061 & 61 \\
\hline $\mathrm{H}(21 \mathrm{~A})$ & 6902 & 12876 & 8818 & 21 \\
\hline $\mathrm{H}(21 \mathrm{~B})$ & 7004 & 13270 & 9311 & 21 \\
\hline
\end{tabular}




\begin{tabular}{lrrrr}
$\mathrm{H}(23)$ & 5105 & 14094 & 8302 & 25 \\
$\mathrm{H}(24)$ & 5083 & 15783 & 8034 & 29 \\
$\mathrm{H}(25)$ & 6218 & 17105 & 8453 & 32 \\
$\mathrm{H}(26)$ & 7389 & 16749 & 9134 & 32 \\
$\mathrm{H}(27)$ & 7414 & 15063 & 9398 & 24 \\
$\mathrm{H}(31 \mathrm{~A})$ & 9974 & 10646 & 9886 & 19 \\
$\mathrm{H}(31 \mathrm{~B})$ & 9467 & 9739 & 10210 & 19 \\
$\mathrm{H}(32)$ & 7950 & 11529 & 10200 & 13 \\
$\mathrm{H}(33 \mathrm{~A})$ & 7216 & 10572 & 10798 & 17 \\
$\mathrm{H}(33 \mathrm{~B})$ & 5552 & 10683 & 10535 & 17 \\
$\mathrm{H}(35)$ & 4652 & 9100 & 10065 & 18 \\
$\mathrm{H}(36)$ & 4529 & 7310 & 10043 & 24 \\
$\mathrm{H}(37)$ & 6231 & 6331 & 10493 & 31 \\
$\mathrm{H}(38)$ & 8106 & 7140 & 10954 & 27 \\
$\mathrm{H}(39)$ & 8235 & 8935 & 10980 & 21 \\
& & & & \\
\hline
\end{tabular}


Table 6. Torsion angles $\left[^{\circ}\right]$ for WY 111403.

\begin{tabular}{|c|c|c|c|}
\hline C(13)-Si-O(1)-C(7) & $143.9(2)$ & $\mathrm{C}(13)-\mathrm{Si}-\mathrm{C}(15)-\mathrm{C}(16)$ & $69.3(3)$ \\
\hline $\mathrm{C}(15)-\mathrm{Si}-\mathrm{O}(1)-\mathrm{C}(7)$ & $-98.3(3)$ & $\mathrm{C}(11)-\mathrm{Si}-\mathrm{C}(15)-\mathrm{C}(16)$ & $-168.1(3)$ \\
\hline $\mathrm{C}(11)-\mathrm{Si}-\mathrm{O}(1)-\mathrm{C}(7)$ & $23.8(3)$ & $\mathrm{C}(2)-\mathrm{O}(2)-\mathrm{C}(21)-\mathrm{C}(22)$ & $170.8(2)$ \\
\hline $\mathrm{C}(30)-\mathrm{N}-\mathrm{C}(1)-\mathrm{O}(3)$ & $174.7(3)$ & $\mathrm{O}(2)-\mathrm{C}(21)-\mathrm{C}(22)-\mathrm{C}(27)$ & $116.6(3)$ \\
\hline $\mathrm{C}(32)-\mathrm{N}-\mathrm{C}(1)-\mathrm{O}(3)$ & $-9.1(4)$ & $\mathrm{O}(2)-\mathrm{C}(21)-\mathrm{C}(22)-\mathrm{C}(23)$ & $-66.9(4)$ \\
\hline $\mathrm{C}(30)-\mathrm{N}-\mathrm{C}(1)-\mathrm{C}(2)$ & $-9.3(5)$ & $C(27)-C(22)-C(23)-C(24)$ & $-0.1(5)$ \\
\hline $\mathrm{C}(32)-\mathrm{N}-\mathrm{C}(1)-\mathrm{C}(2)$ & $166.9(3)$ & $\mathrm{C}(21)-\mathrm{C}(22)-\mathrm{C}(23)-\mathrm{C}(24)$ & $-176.6(3)$ \\
\hline $\mathrm{C}(21)-\mathrm{O}(2)-\mathrm{C}(2)-\mathrm{C}(3)$ & $-174.0(2)$ & $C(22)-C(23)-C(24)-C(25)$ & $-0.1(5)$ \\
\hline $\mathrm{C}(21)-\mathrm{O}(2)-\mathrm{C}(2)-\mathrm{C}(1)$ & $69.5(3)$ & $C(23)-C(24)-C(25)-C(26)$ & $0.2(5)$ \\
\hline $\mathrm{O}(3)-\mathrm{C}(1)-\mathrm{C}(2)-\mathrm{O}(2)$ & $39.8(4)$ & $C(24)-C(25)-C(26)-C(27)$ & $-0.1(5)$ \\
\hline $\mathrm{N}-\mathrm{C}(1)-\mathrm{C}(2)-\mathrm{O}(2)$ & $-136.0(3)$ & $C(23)-C(22)-C(27)-C(26)$ & $0.2(5)$ \\
\hline $\mathrm{O}(3)-\mathrm{C}(1)-\mathrm{C}(2)-\mathrm{C}(3)$ & $-75.8(4)$ & $\mathrm{C}(21)-\mathrm{C}(22)-\mathrm{C}(27)-\mathrm{C}(26)$ & $176.7(3)$ \\
\hline $\mathrm{N}-\mathrm{C}(1)-\mathrm{C}(2)-\mathrm{C}(3)$ & 108.3(3) & $\mathrm{C}(25)-\mathrm{C}(26)-\mathrm{C}(27)-\mathrm{C}(22)$ & $-0.1(5)$ \\
\hline $\mathrm{O}(2)-\mathrm{C}(2)-\mathrm{C}(3)-\mathrm{O}(5)$ & $-167.1(2)$ & $\mathrm{C}(31)-\mathrm{O}(4)-\mathrm{C}(30)-\mathrm{N}$ & $-4.5(3)$ \\
\hline $\mathrm{C}(1)-\mathrm{C}(2)-\mathrm{C}(3)-\mathrm{O}(5)$ & $-50.1(3)$ & $\mathrm{C}(31)-\mathrm{O}(4)-\mathrm{C}(30)-\mathrm{S}$ & $177.0(2)$ \\
\hline $\mathrm{O}(2)-\mathrm{C}(2)-\mathrm{C}(3)-\mathrm{C}(4)$ & $65.0(3)$ & $\mathrm{C}(1)-\mathrm{N}-\mathrm{C}(30)-\mathrm{O}(4)$ & $164.4(3)$ \\
\hline$C(1)-C(2)-C(3)-C(4)$ & $-178.1(2)$ & $\mathrm{C}(32)-\mathrm{N}-\mathrm{C}(30)-\mathrm{O}(4)$ & $-12.0(3)$ \\
\hline $\mathrm{O}(5)-\mathrm{C}(3)-\mathrm{C}(4)-\mathrm{C}(5)$ & $74.5(3)$ & $\mathrm{C}(1)-\mathrm{N}-\mathrm{C}(30)-\mathrm{S}$ & $-17.2(5)$ \\
\hline $\mathrm{C}(2)-\mathrm{C}(3)-\mathrm{C}(4)-\mathrm{C}(5)$ & $-159.3(3)$ & $\mathrm{C}(32)-\mathrm{N}-\mathrm{C}(30)-\mathrm{S}$ & $166.4(2)$ \\
\hline $\mathrm{O}(5)-\mathrm{C}(3)-\mathrm{C}(4)-\mathrm{C}(6)$ & $-50.1(3)$ & $\mathrm{C}(30)-\mathrm{O}(4)-\mathrm{C}(31)-\mathrm{C}(32)$ & $18.3(3)$ \\
\hline $\mathrm{C}(2)-\mathrm{C}(3)-\mathrm{C}(4)-\mathrm{C}(6)$ & $76.1(3)$ & $\mathrm{C}(30)-\mathrm{N}-\mathrm{C}(32)-\mathrm{C}(31)$ & $21.8(3)$ \\
\hline$C(5)-C(4)-C(6)-C(7)$ & $78.0(3)$ & $\mathrm{C}(1)-\mathrm{N}-\mathrm{C}(32)-\mathrm{C}(31)$ & $-155.2(3)$ \\
\hline $\mathrm{C}(3)-\mathrm{C}(4)-\mathrm{C}(6)-\mathrm{C}(7)$ & $-158.3(3)$ & $\mathrm{C}(30)-\mathrm{N}-\mathrm{C}(32)-\mathrm{C}(33)$ & $-101.5(3)$ \\
\hline $\mathrm{Si}-\mathrm{O}(1)-\mathrm{C}(7)-\mathrm{C}(8)$ & $-160.2(2)$ & $\mathrm{C}(1)-\mathrm{N}-\mathrm{C}(32)-\mathrm{C}(33)$ & $81.6(3)$ \\
\hline $\mathrm{Si}-\mathrm{O}(1)-\mathrm{C}(7)-\mathrm{C}(6)$ & $78.1(3)$ & $\mathrm{O}(4)-\mathrm{C}(31)-\mathrm{C}(32)-\mathrm{N}$ & $-22.9(3)$ \\
\hline $\mathrm{C}(4)-\mathrm{C}(6)-\mathrm{C}(7)-\mathrm{O}(1)$ & $-162.7(3)$ & $\mathrm{O}(4)-\mathrm{C}(31)-\mathrm{C}(32)-\mathrm{C}(33)$ & $98.3(3)$ \\
\hline$C(4)-C(6)-C(7)-C(8)$ & $78.4(3)$ & $\mathrm{N}-\mathrm{C}(32)-\mathrm{C}(33)-\mathrm{C}(34)$ & $74.7(3)$ \\
\hline $\mathrm{O}(1)-\mathrm{Si}-\mathrm{C}(11)-\mathrm{C}(12)$ & $175.2(2)$ & $\mathrm{C}(31)-\mathrm{C}(32)-\mathrm{C}(33)-\mathrm{C}(34)$ & $-39.1(4)$ \\
\hline$C(13)-S i-C(11)-C(12)$ & $58.8(3)$ & $\mathrm{C}(32)-\mathrm{C}(33)-\mathrm{C}(34)-\mathrm{C}(35)$ & $-75.7(4)$ \\
\hline C(15)-Si-C(11)-C(12) & $-62.7(3)$ & $\mathrm{C}(32)-\mathrm{C}(33)-\mathrm{C}(34)-\mathrm{C}(39)$ & $102.1(3)$ \\
\hline $\mathrm{O}(1)-\mathrm{Si}-\mathrm{C}(13)-\mathrm{C}(14)$ & $-68.2(3)$ & $\mathrm{C}(39)-\mathrm{C}(34)-\mathrm{C}(35)-\mathrm{C}(36)$ & $-0.8(5)$ \\
\hline C(15)-Si-C(13)-C(14) & $173.4(3)$ & $\mathrm{C}(33)-\mathrm{C}(34)-\mathrm{C}(35)-\mathrm{C}(36)$ & 177.1(3) \\
\hline C(11)-Si-C(13)-C(14) & $51.4(3)$ & $\mathrm{C}(34)-\mathrm{C}(35)-\mathrm{C}(36)-\mathrm{C}(37)$ & $0.7(5)$ \\
\hline $\mathrm{O}(1)-\mathrm{Si}-\mathrm{C}(15)-\mathrm{C}(16)$ & $-45.8(3)$ & $\mathrm{C}(35)-\mathrm{C}(36)-\mathrm{C}(37)-\mathrm{C}(38)$ & $-0.6(5)$ \\
\hline
\end{tabular}


$\mathrm{C}(36)-\mathrm{C}(37)-\mathrm{C}(38)-\mathrm{C}(39)$

Symmetry transformations used to generate equivalent atoms:

Table 7. Hydrogen bonds for WY111403 [ $\AA$ and $\left.{ }^{\circ}\right]$.

\begin{tabular}{lcccc}
\hline D-H...A & d(D-H) & d(H...A & $d($ D..A $)$ & $<$ (DHA) \\
\hline O(5)-HO5...S & 0.82 & 2.52 & $3.282(2)$ & 154.4
\end{tabular}

Symmetry transformations used to generate equivalent atoms: 